\title{
CHAIM SAMUEL HÖNIG (1926 - 2018): TRAJETÓRIA ACADÊMICA E CONTRIBUIÇÕES PARA O DESENVOLVIMENTO DA MATEMÁTICA BRASILEIRA ${ }^{1}$
}

\author{
Mariana Feiteiro Cavalari - Universidade Federal de Itajubá - UNIFEI - Brasil \\ Sergio Roberto Nobre - UNESP Câmpus Rio Claro - Brasil
}

(aceito para publicação em maio de 2019)

\begin{abstract}
Resumo
Esta pesquisa teve como objetivo analisar a atuação acadêmica do professor Chaim Samuel Hönig (1926-2018) para o desenvolvimento da Matemática no Brasil. Para tanto, investigamos sua trajetória acadêmica privilegiando suas principais obras e suas contribuições para o desenvolvimento da Comunidade Matemática brasileira. O professor Chaim trabalhou na Universidade de São Paulo, por mais de quatro décadas, lecionando na graduação e na pós-graduação. Durante sua carreira acadêmica, realizou pesquisas em variadas áreas da Matemática e, dentre suas principais produções, destacamos três livros da área de Análise amplamente utilizados para a formação de matemáticos no Brasil. O professor Chaim teve atuação destacada na criação e ampliação dos canais de comunicação entre os matemáticos brasileiros. Foi idealizador dos Colóquios Brasileiros de Matemática e coordenador da primeira edição deste evento. Foi membro fundador da Sociedade Brasileira de Matemática (SBM), o primeiro presidente desta sociedade e, ainda, o primeiro editor chefe do Boletim da SBM. Nas décadas de 1960 e 1970 foi membro da comissão organizadora de vários eventos científicos, em especial, na área de Análise. Destacamos sua participação na criação dos Seminários Brasileiros de Análise e na organização de suas primeiras edições. Devido ao fato de este docente ter atuado na formação de matemáticos, ter elaborado manuais científicos e ter auxiliado na ampliação dos canais de comunicação entre os matemáticos no Brasil, podemos afirmar que o professor Chaim contribuiu para o desenvolvimento da Comunidade Matemática em território nacional.
\end{abstract}

Palavras-Chave: Matemática, História, Biografia, Século XX, Comunidade Matemática Brasileira.

\footnotetext{
${ }^{1}$ Este artigo apresenta resultados da tese intitulada "As contribuições de Chaim S. Hönig para o desenvolvimento da Matemática Brasileira" realizada sob a orientação do Prof. Dr. Sérgio Roberto Nobre no Programa de PósGraduação em Educação Matemática da Universidade Estadual Júlio de Mesquita Filho - UNESP câmpus Rio Claro. Alguns resultados apresentados neste artigo estão presentes nos trabalhos intitulados "Uma narrativa da trajetória acadêmica de Chaim Samuel Hönig (1926 - )", "Chaim Samuel Hönig (1926- ) e a Comunidade Matemática Brasileira", "Descendência Científica de Chaim Samuel Hönig (1926 - )" divulgados nos anais do Seminário Nacional de História da Ciência de 2012 e 2016 e do Seminário Nacional de História da Matemática de 2013, respectivamente.
}

RBHM, Vol. 19, nº 37, p. 117-139, 2019 


\title{
CHAIM SAMUEL HöNIG (1926 - 2018): ACADEMIC CAREER AND CONTRIBUTIONS TO THE DEVELOPMENT OF BRAZILIAN MATHEMATICS
}

\begin{abstract}
This research aims at analyzing Professor Chaim S. Hönig's (1926 - 2018) contributions to the development of Brazilian Mathematics. In order to achieve this goal, his academic career is investigated, with emphasis to his main works and his contributions to the creation and development of the Brazilian Community of Mathematics. Professor Chaim worked at Universidade de Sao Paulo, for over four decades, teaching at both undergraduate and graduate levels. During his academic career, he carried out research in several fields of Mathematics and among his main academic works, we highlight three books in the Analysis field, which are broadly used in the teaching of mathematicians in Brazil. Professor Chaim had a distinguished role for the improvement of communication among scholars who carried out research in Mathematics in the national territory, was a member and a founder of the Sociedade Brasileira de Matemática (Brazilian Society of Mathematics) - (SBM), was the first president to that association and was the first chief editor of SBM's journal as well. During the 1960's and 1970's, he was a member of the commission in charge of the organization of various scientific events, mainly in the area of Analysis. His participation was significant in the creation of the first editions of the Seminários Brasileiros de Análise (Brazilian Seminars of Analysis). Because this scholar has participated in the education of mathematicians, elaborated scientific manuals, and helped make communication amongst mathematicians in Brazil more effective, it can be affirmed that Professor Hönig has contributed to the development of the Community of Mathematics in Brazil.
\end{abstract}

Key-words: Mathematics, History, Biography, Century XX, Brazilian Mathematical Community

\section{Introduçao}

Os primeiros núcleos de pesquisa em Matemática no Brasil começaram a se constituir, nos anos 1930, com a criação da Faculdade de Filosofia Ciências e Letras (FFCL) de São Paulo, em 1934 e da Escola de Ciências da Universidade do Distrito Federal (UDF), em 1935.

Hönig e Gomide (1976) utilizam o termo "pré-história" da Matemática para designar o período anterior à criação destas faculdades/escolas, não pelo fato de não existir ensino de Matemática neste período no Brasil, mas porque seu ensino estava ligado ao militarismo ou a escolas de engenharia, desta forma não existia, efetivamente, uma Comunidade Matemática.

Na década posterior a criação dos cursos de Matemática da FFCL de São Paulo e da UDF, foram iniciados cursos de Matemática em instituições de ensino superior, públicas e privadas, tais como, a Universidade de Porto Alegre, a Universidade Católica de São Paulo, a Universidade Presbiteriana Mackenzie e a Universidade Católica de Campinas, bem como, as Faculdades de Filosofia do Paraná, da Bahia e de Minas Gerais. 
No final da década de 1940, embora não existissem programas de pós-graduação institucionalizados ${ }^{2}$, começam-se, nestas instituições, a ter defesas de teses na área de Ciências Matemáticas e, assim, tem-se, nestes centros, doutores em Matemática.

Os matemáticos brasileiros, no decorrer dos anos 1940, 50 e 60, passaram a se organizar para a criação de sociedades, periódicos científicos e, também, de centros de pesquisas que promoveram a produção e a divulgação científica, em Matemática, em território nacional. Neste contexto, merece destaque a criação da Sociedade Brasileira para o Progresso da Ciência - SBPC (em 1948), da Sociedade de Matemática de São Paulo - SMSP (em 1945), da Sociedade Brasileira de Matemática - SBM (em 1969), do Centro Brasileiro de Pesquisas Físicas - CBPF (em 1949) e do Instituto de Matemática Pura e Aplicada - IMPA (em 1952). Enfatizamos também a publicação do periódico Summa Brasiliensis Mathematicae (em 1945) e do Boletim da SMSP (em 1946). Merece ênfase ainda, que desde o início do século XX, já existia a Academia Brasileira de Ciências, que promovia a publicação periódica dos "Anais da Academia Brasileira de Ciências", nos quais podemos encontrar artigos matemáticos.

Além disto, a criação de programas de bolsistas para doutoramento no exterior e de agências de fomento, tais como, o Conselho Nacional de Pesquisa - CNPq e a Campanha de Aperfeiçoamento de Pessoal de Ensino Superior- CAPES ${ }^{3}$, no início da década de 1950, estimulou os brasileiros a realizarem pesquisas científicas (HÖNIG, GOMIDE, 1976).

Neste período, de acordo Hönig e Gomide (1976), foram ampliados os programas de professores visitantes e as bibliotecas nos grandes centros de pesquisa e, assim, houve condições para a "grande expansão" da pesquisa em Matemática que se iniciou nos anos 1950 e se desenvolveu nos anos 1970.

De fato, nos anos 1950, 1960 e 1970 houve uma expansão de cursos de graduação em Matemática e de institutos nos quais foram criados grupos de pesquisa em Matemática, por exemplo, a FFCL de Rio Claro (posteriormente UNESP), a Universidade de São Paulo USP câmpus São Carlos, a Universidade Estadual de Campinas - UNICAMP, a Universidade Federal de Pernambuco - UFPE, a Universidade Federal do Paraná, a Universidade de Brasília - UnB e o Instituto Tecnológico de Aeronáutica - ITA.

Na década de 1960, concomitantemente com a criação de programas de pósgraduação institucionalizados, os matemáticos brasileiros já estavam inseridos "[...] no contexto científico internacional e [... elaboravam] artigos de alta qualidade publicados nas mais importantes revistas internacionais" (SILVA, AZEVEDO, 2005, s/p).

Foi neste período que teve influência o matemático Chaim Samuel Hönig, que atuou na USP por mais de 40 anos e teve destacada atuação na organização de importantes eventos matemáticos no Brasil, além de ter sido membro fundador e primeiro presidente da SBM.

\footnotetext{
${ }^{2}$ Neste período, a pós-graduação era regulamentada pelo decreto n. 12.511 de 1942 , que em seu artigo $64^{\circ}$ definia que seria concedido o título de doutor ao bacharel que defendesse tese de "notável valor", depois de pelo menos dois anos de estudos sob a orientação de um professor catedrático da disciplina relativa ao seu trabalho e que fosse aprovado no exame de duas disciplinas da mesma seção ou de seção afim. Um graduado, também, poderia receber o título de doutor se fosse aprovado em um concurso para professor catedrático (SILVA, 2003).

3 Atualmente são, respectivamente, denominados "Conselho Nacional de Desenvolvimento Científico e Tecnológico" e "Coordenação de Aperfeiçoamento de Pessoal de Ensino Superior".
}

RBHM, Vol. 19, n 37, p. 117-139, 2019 
Diante destas considerações, entendemos ser relevante a realização de uma investigação com foco na biografia deste acadêmico.

A importância da realização de trabalhos voltados para a produção de biografias de personagens de destaque para o desenvolvimento da Matemática no Brasil foi enfatizada por Baroni e Nobre (1999). Estes autores, também ressaltam, que, no final dos anos 1990, o campo de pesquisa relativo à biografia de matemáticos no Brasil, estava "[...] totalmente aberto e inexplorado" (p. 131).

Nos anos 2000, este cenário começou a se alterar, quando foram elaborados alguns trabalhos acadêmicos com o objetivo de narrar trajetórias pessoais e acadêmicas de Matemáticos brasileiros, dentre os quais citamos, Badin (2006), Calabria (2015), Cobra (2014), Duarte (2014) e Souto (2006). Assim, podemos afirmar que trabalhos sobre biografias de Matemáticos brasileiros começam a ganhar espaço no âmbito de pesquisas em História da Matemática em nosso país.

Neste contexto, cumpre destacar que a atuação de muitos matemáticos brasileiros, inclusive do professor Chaim, não é relevante no cenário matemático internacional, entretanto, é relevante no âmbito nacional. D'Ambrosio (2003) afirma que, podemos identificar no Brasil, “[...] nomes que não tiveram e não terão importância na história da ciência como um todo. A importância de se identificar e analisar [...] estes nomes está no entender a evolução do pensamento brasileiro, das nossas idéias e nossas instituições" (p. vii)

Nesta perspectiva de auxiliar a compreender o desenvolvimento da Matemática brasileira e desta forma, contribuir para a escrita da História desta área do conhecimento no Brasil que foi realizada esta investigação com foco na biografia do Professor Chaim.

Consideramos que uma biografia é a narrativa de uma representação da trajetória de uma determinada personagem e desta maneira, não é capaz de "[...] expressar os inúmeros, descontínuos e contraditórios fios de um destino pessoal”. (SCHMIDT, 2004, p. 132). Assim, ressaltamos a necessidade de, em uma pesquisa biográfica, explicitar quais aspectos da vida do biografado serão investigados. No caso da presente investigação, temos como foco a trajetória profissional deste docente, destacando suas principais obras, sua descendência cientifica e suas contribuições para o desenvolvimento da Comunidade Matemática brasileira.

Para tanto, dividimos o presente artigo em seções, na primeira apresentamos os caminhos percorridos por esta investigação, posteriormente, apresentamos dados biográficos do Professor Chaim, bem como considerações sobre algumas de suas obras matemáticas e por fim, apresentamos a seção destinada a contribuições deste docente para o desenvolvimento da Comunidade Matemática no Brasil.

\section{Caminhos percorridos no desenvolvimento da investigação}

Para a realização dessa investigação, inicialmente, consultamos duas biografias sucintas do docente Chaim (SILVA, 2008 e ABC, s/d), um memorial matemático do Professor Chaim datado de 1959, um currículo deste docente relativo ao ano de 1991 e uma entrevista do professor Chaim realizada pelo Professor Possani em 2003.

Contatamos por correio eletrônico e realizamos entrevistas com alguns professores que tiveram contato com o professor Chaim e/ou com seus orientados. Além disto, realizamos 
três entrevistas com o Professor Chaim ${ }^{4}$. Para as entrevistas foram elaborados roteiros, parcialmente abertos, com base em informações obtidas em fontes consultadas anteriormente.

De acordo com Lacouture (1998), a utilização de relatos, depoimentos, é inerente ao processo de escrita da história imediata (e consequentemente, do tempo presente) que é elaborada a partir dos arquivos vivos, que são os homens e mulheres que foram atores desta história.

Os depoimentos destes atores são realizados com base nas lembranças e, de acordo com Bosi (1987), “[...] lembrar não é reviver, mas refazer, reconstruir, repensar, com imagens e ideias de hoje, as experiências do passado [...]" (p. 17). Neste sentido, cientes das limitações da lembrança, as informações obtidas nos depoimentos orais, como nos outros documentos históricos utilizados na presente investigação, foram analisadas e confrontadas.

Destacamos que conhecer e entrevistar o professor Chaim no desenvolvimento desta investigação, por um lado nos possibilitou o acesso a informações que possivelmente não ficariam registradas na forma escrita, mas por outro lado, trouxe algumas limitações a investigação.

Com relação as limitações, podemos citar que identificamos, nos depoimentos do professor Chaim, um tipo de "censura" que o inibia de contar episódios que pudesse chatear algum colega. Esta pode ser evidenciada pelo seguinte trecho da entrevista com este docente realizada em 2010: "[...] não vou citar nomes, porque pode omitir alguém, o Schwartz não tem problema, pois tem medalha Fields, então ok, ninguém pode se chatear por citar o Schwartz e não o citar." (Informação verbal). A presença desta censura na narrativa do professor Chaim dificultou a busca de dados da presente investigação e exigiu um maior cuidado na análise das entrevistas.

Além disto, destacamos que, devido a sua personalidade reservada, o professor Chaim não nos forneceu fotos, documentos pessoais e tampouco manuscritos de trabalhos científicos ${ }^{5}$.

Devido à estas dificuldades tivemos que encontrar alternativas para investigar a produção acadêmica do professor Chaim. Com o intuito de estudar a obra matemática deste docente, analisamos informações disponíveis em um memorial acadêmico do Professor Chaim (1959), no currículo vitae deste docente (1991) e nos bancos de dados das bibliotecas das Universidades Estaduais Paulistas. Já para identificar a descendência cientifica do Professor Chaim, analisamos as informações obtidas junto aos docentes contatados, em uma lista de teses e dissertações defendidas no IME-USP (2005), no currículo vitae do Professor Chaim de 1991, em Badin (2006), em Azevedo \& Silva (s/d) e em variados Currículos Vitae disponíveis na plataforma Lattes.

\footnotetext{
${ }^{4}$ Esta biografia foi produzida em um momento que o Prof. Chaim ainda estava vivo e, embora seja mais comum a produção de biografias de personalidades já falecidas, Nobre (2000) destaca a existência de textos biográficos de pessoas ligadas a ciência que foram escritos quando estas ainda se encontravam vivas, e afirma que estes textos "[...] trouxeram enormes contribuições à historiografia da Matemática" (p. 11).

${ }^{5}$ Decidimos, devido ao fato do professor Chaim estar vivo no desenvolvimento da investigação, não solicitar documentações aos familiares.
}

RBHM, Vol. 19, nº 37, p. 117-139, 2019 
Neste sentido, podemos afirmar que a dificuldade para localizar fontes históricas relativas ao nosso objeto de pesquisa, impôs limitações ao desenvolvimento da investigação que se refletiu em "lacunas" históricas neste texto.

Assim, a presente pesquisa pode ser entendida como uma primeira versão da trajetória acadêmica do Professor Chaim S. Hönig e, esta pode contribuir para a escrita de uma história do desenvolvimento da Matemática no Brasil e da criação da Comunidade Matemática brasileira.

\section{Uma biografia de Chaim Samuel Hönig}

Chaim Samuel Hönig, filho primogênito de Moisés E. Hönig e Rachela Hönig, nasceu em $1^{\circ}$ de fevereiro de 1926, em Berlim na Alemanha. Moisés e Rachela eram judeus de origem polonesa e, em 1937, devido à ascensão do nazismo decidiram se mudar, com seus filhos, da Alemanha.

Neste período, de acordo com o professor Chaim em entrevista em 2010, poucos países estavam recebendo imigrantes alemães. O Brasil, por exemplo, somente asilava pessoas que possuíssem parentes em território nacional. Como o tio de Chaim, Donleistner, residia em Porto Alegre - RS desde a década de 1920, a família Hönig decidiu se mudar para o Brasil e se radicar na capital do Rio Grande do Sul.

Chaim, de acordo com entrevista concedida a nós em 2010, iniciou seus estudos na Alemanha e desde a infância, incentivado pelo seu pai, se interessou pela Matemática. No Brasil, cursou o Ginásio e o Secundário no Colégio Estadual Júlio de Castilhos. Durante o ensino secundário, por se destacar nos estudos, o seu professor de Matemática, indicou que ele, concomitantemente, com o secundário assistisse, como ouvinte, a cursos de Matemática lecionados pelos professores Antônio Rodrigues (1918-2003) e Ary Nunes Tietböhl (19121998) na Faculdade de Educação, Ciências e Letras de Porto Alegre.

De acordo com o professor Chaim, em entrevista em 2010, assistir estes cursos foi fundamental para sua carreira. Afinal, embora tivesse facilidade e interesse em Matemática desde criança, ele desconhecia a existência de instituições nas quais fosse possível estudar exclusivamente Matemática. Além disto, os citados professores tinham se graduado na FFCL da USP e foi por intermédio deles que Chaim soube do curso de graduação em Matemática da FFCL- USP.

Em 1946, Chaim foi aprovado no exame de vestibular desta faculdade. Cursou, concomitantemente, o Bacharelado em Física e em Matemática aos quais concluiu em 1948 e no ano seguinte licenciou-se em Matemática pela mesma instituição.

Na graduação foi aluno dos professores Cândido Lima da Silva Dias (1913-1998), André Weil (1906-1998), Jean A. E. Dieudonné (1906-1992) e Jean F. A. Delsarte (19031968). Assim, podemos identificar que, na formação inicial, o professor Chaim teve contato com matemáticos bourbakistas.

Em 1951, de acordo com o professor Chaim em entrevista em 2010, ele iniciou por indicação do professor Cândido Dias, sua carreira docente na FFCL, como primeiro 
assistente $^{6}$ do professor Edison Farah (1915-2006) na cadeira de Análise Superior e, desta forma, responsabilizou-se pelas aulas de exercícios.

No âmbito da Cátedra de Análise Superior, o professor Chaim, realizou sua pesquisa de doutoramento. Em 1952, obteve o Título de Doutor em Ciências (Matemática), na USP São Paulo, por meio da defesa da tese intitulada "Sôbre um método de refinamento de Topologias", realizado, oficialmente, sob a orientação de Edison Farah. De acordo com o professor Chaim, em entrevista concedida a nós em 2008, embora o orientador oficial tenha sido o professor Farah, esta tese teve grande influência do matemático Leopoldo Nachbin (1922-1993).

O referido trabalho foi aprovado com distinção pela banca examinadora constituída pelos professores Edison Farah, Omar Catunda (1906-1986) e Cândido Lima da Silva Dias da FFCL de São Paulo e por Leopoldo Nachbin do CBPF e Charles Ehresmann (1905-1979) da Universidade de Strasbourg.

O professor Chaim realizou pós-doutorado no Institut Henri Poincarè na França, no período de 1953 a 1955, com bolsa do CNPq e do Centre National de la Recherche Scientifique (CNRS). Segundo este docente, em entrevista em 2010, o contato com o referido Instituto foi mediado pelo Professor Cândido Dias e a decisão de estudar na França foi pautada em sua formação de influência bourbakista na FFCL. Neste Instituto, teve a oportunidade de conhecer diversos pesquisadores, dos quais destacou Laurent Schwartz (1915-2002), que recentemente havia sido laureado com a medalha Fields?.

Após retornar ao Brasil, Chaim voltou a lecionar como assistente do professor Farah, na cadeira de Análise Superior. Posteriormente, de acordo com o Professor Chaim em entrevista em 2010, no ano letivo de 1959, a convite do professor L. Nachbin, trabalhou simultaneamente, no IMPA ${ }^{8}$ e na USP.

Neste mesmo ano, o professor Chaim elaborou a tese intitulada "Sôbre uma generalização dos números Reais e a sua aplicação na classificação dos grupos sem torção" para um concurso que seria realizado na Faculdade Nacional de Filosofia, Ciências e Letras da Universidade do Brasil. Entretanto, de acordo com o professor Chaim, em entrevista em 2010, este concurso não se realizou.

No início da década de 1960, foi contratado como assistente do professor Mário Schenberg (1914-1990), catedrático de Mecânica Racional e Mecânica Celeste na FFCL de São Paulo.

Trabalhou, de 1961 a 1964, como professor visitante da Faculté des Sciences em Rennes na França. Teve, então, a oportunidade de encontrar-se, novamente, com L. Schwartz e de participar, periodicamente, de seminários Matemáticos realizados em Paris.

\footnotetext{
${ }^{6} \mathrm{O}$ doutoramento era um aprofundamento dos estudos, realizado pelos professores assistentes, em um assunto relativo a área de sua disciplina, sob a supervisão do catedrático (CASTRUCCI, 1993).

${ }^{7}$ De acordo com o professor Alexandre Rodrigues, em entrevista concedida a nós em 2010, a realização de estudos pós-graduados de docentes da FFCL em instituições estrangeiras, foi importante para o desenvolvimento da Matemática na USP, pois estes pesquisadores ao retornarem ao Brasil, compartilhavam com seus colegas novas obras e as tendências da pesquisa em Matemática em outros países.

${ }^{8}$ De acordo com o Professor Chaim em entrevista em 2010, ele formalmente fora contratado para lecionar no CBPF, mas efetivamente, trabalhou no IMPA.
}

RBHM, Vol. 19, nº 37, p. 117-139, 2019 
Embora seu contrato na França vencesse somente na metade de 1964, Chaim decidiu retornar ao Brasil antes do início do ano letivo. Chegou em território nacional no final de março de 1964 e, segundo este professor, em entrevista em 2011, se tivesse esperado o término do contrato, devido à instauração da ditadura militar, possivelmente não teria retornado ao Brasil.

De fato, este período foi muito tumultuado nas universidades brasileiras e, consequentemente, na USP. No entanto, de acordo com o professor Chaim em entrevista concedida a nós em 2011, não houve repressão no IME. Segundo ele: “[...] tinham pessoas que tentavam usar o período militar espalhavam boatos, que iam pegar aquele e que iam caçar este, no Instituto [IME] que eu saiba nada disto se realizou. [...]” (Informação verbal).

Ainda no ano letivo de 1964, Chaim assumiu a regência, como professor contratado, da recém-criada cadeira de Equações Diferenciais a qual regeu até a Reforma Universitária. A partir de então passou a se responsabilizar essencialmente, por disciplinas da área de Análise, Análise Funcional e cursos de Álgebra Linear.

A docência sempre foi uma importante atividade em sua carreira acadêmica e era, de acordo com D’Ambrosio, em entrevista concedida a nós em 2009, um professor “[...] muito cuidadoso, levava muito a sério o trabalho dele de dar aulas, aula muito ordenada, uma pessoa muito bacana" (informação verbal).

Além da organização, os alunos do professor Chaim ressaltam que em suas aulas este docente se mostrava muito metódico e rigoroso. $\mathrm{O}$ procedimento de avaliação utilizado nos cursos era constituído de várias "provinhas" que eram realizadas no decorrer do semestre. Este procedimento, de acordo com o professor Chaim, em entrevista ao professor Possani em 2003, obrigava o aluno a estudar durante todo o curso e, desta forma, aumentava significativamente o índice de aprovação.

O professor Chaim estruturava seus cursos introdutórios de modo a sempre apresentar ao estudante aplicações dos conteúdos matemáticos abordados. Segundo ele, em entrevista ao Professor Possani em 2003, esta estrutura é interessante, devido ao fato de que muitos alunos compreendem um teorema somente após conhecer uma aplicação deste.

Em 1965, defendeu na FFCL da USP a tese intitulada "Análise de Fourier em Espaços 1 e Teoremas do Tipo Sobolev" e obteve o título de Professor Livre-Docente. Após oito anos, por meio de concurso, tornou-se Professor Titular em Matemática da USP, cargo no qual se aposentou compulsoriamente em 1996, ao completar setenta anos.

Durante sua carreira acadêmica, o professor Chaim participou de vários congressos e proferiu diversas conferências matemáticas no Brasil, na Alemanha, na França, na Polônia e na antiga Tchecoslováquia. Além disto, realizou dez estágios em universidades estrangeiras.

Atuou, também, em comissões organizadoras de importantes eventos matemáticos em território nacional. Foi o idealizador e coordenador do I Colóquio Brasileiro de Matemática (CBM), o primeiro evento, de abrangência nacional, que congregava exclusivamente matemáticos no Brasil ${ }^{9}$. Esse Colóquio foi realizado na cidade Poços de

\footnotetext{
${ }^{9}$ É necessário ressaltar que, de acordo com o professor Chaim em entrevista em 2010, no ano de 1952, foi realizada, no ITA, uma reunião de pessoas interessadas em pesquisas na área de Matemática, que contou com a participação de cerca de 10 acadêmicos. Entretanto, este evento não teve continuidade.
} 
Caldas-MG, no Palace Hotel, no período de $1^{\circ}$. a 20 de julho de 1957 e contou oficialmente com a participação de 49 acadêmicos, sendo dois estrangeiros e 47 brasileiros provenientes de nove centros universitários brasileiros.

De acordo com o professor Chaim, em entrevista concedida a nós em 2008, no início da década de 1950, existiam poucos professores interessados pela "Matemática Moderna". Esta seria a Matemática nos moldes introduzidos pelos professores estrangeiros na FFCL, que se distanciava daquela Matemática que ensinada nas escolas de engenharia.

Segundo este docente, entrevista concedida a nós em 2008, em 1956, durante a $8^{\mathrm{a}}$ Reunião anual da SBPC, realizada em Ouro Preto, diversos pesquisadores se mostraram interessados em conceitos relativos à "Álgebra moderna" e "Topologia Geral”. Diante disso, o professor Chaim teve a ideia de criar um encontro cujo objetivo seria divulgar os resultados das pesquisas em "Matemática moderna" realizadas no país, além de reunir e atrair novos talentos para esta área que ainda era muito recente em território nacional. Após este evento em Minas Gerais, o professor Chaim viajou ao Rio de Janeiro e conversou com Leopoldo Nachbin sobre a ideia da criação de um evento desta natureza. Esta ideia, de acordo com Calábria (2010), também foi debatida com o professor da UFPE, Alfredo Pereira Gomes (1919-2006) que se encontrava no Rio de Janeiro neste período.

Para a organização deste evento, de acordo com o relatório do I CBM, formou-se uma comissão constituída pelo professor Chaim S. Hönig (coordenador) e mais 11 matemáticos de sete instituições brasileiras. O I CBM, em sua programação, previa a realização de palestras e cursos. O Professor Chaim, juntamente com a citada comissão, estabeleceu que os textos que iriam nortear os cursos desses Colóquios deveriam ser redigidos e entregues com antecedência à coordenação do evento, para que pudessem ser copiados e entregues aos participantes dos cursos. De acordo com o professor Chaim, em entrevista concedida a nós em 2011, naquele momento, a produção de um material desta natureza era de extrema importância devido à carência de materiais didáticos de Matemática em língua portuguesa.

Desde 1957, os CBM têm sido realizados, bianualmente, sem interrupções. O professor Chaim participou de diversas edições deste evento e, ainda, atuou na comissão organizadora do II CBM, realizado em 1959, em Poços de Caldas-MG.

No final da década de 1960 e início da de 1970, o professor Chaim, também, participou da comissão organizadora de seis eventos científicos na área de Análise Matemática, a saber: as três "Quinzenas de Análise Funcional e Equações Diferenciais", realizadas no ITA em 1967, 1969 e 1970; o "Simpósio de Análise" realizado, em 1972, na UFPE; a "Semana de Análise Funcional Não-Linear", realizada na UNICAMP, em 1973 e o "Simpósio de Análise Funcional” realizado na UNICAMP em 1974 (TOLEDO, 2008).

Além disto, destacamos a atuação do professor Chaim na organização de diversas edições dos "Seminários Brasileiros de Análise" (SBA). A primeira edição deste evento foi realizada em 1975 e teve a coordenação de Pedro Nowosad, Chaim S. Hönig e Djairo Guedes Figueiredo. Os SBA, de acordo com Toledo (2008), foram criados com o objetivo propiciar o intercâmbio científico entre os analistas brasileiros e se realizam semestralmente. O professor Chaim participou, juntamente com outros docentes, da organização da maioria dos SBA realizados até 2009. 
O professor Chaim, também, coordenou a comissão de organização local da "I Escola Latino-Americana de Matemática", realizada no Rio de Janeiro, em 1968 e foi membro da comissão de organização da "I Escola Latino-Americana de Equações Diferenciais", realizado no IME - USP em 1981. Neste evento, juntamente com o professor Djairo G. Figueiredo, editou e publicou, pela Springer-Verlag, os textos relativos aos cursos proferidos.

Foi membro de importantes sociedades e academias científicas no Brasil. Foi membro titular e fundador da Academia de Ciências do Estado de São Paulo (ACIESP) e foi membro da SMSP, da qual participou da diretoria. Foi, ainda, membro fundador da SBM e seu primeiro presidente. Destacamos que a eleição do Professor Chaim como primeiro presidente da SBM, reforça a relevância de sua atuação na, então, Comunidade Matemática brasileira.

Em 1970, durante seu mandato de presidente da SBM, foi criado o "Boletim da Sociedade Brasileira de Matemática", do qual foi Editor-Chefe. Além deste periódico, de acordo com D'Ambrosio, em entrevista concedida a nós em 2009, o professor Chaim, foi um dos fundadores das "Notas de Matemática e Física". Este periódico, de acordo com D'Ambrosio (1988), foi criado pelos discentes da FFCL da USP, com o apoiop dos docentes desta faculdade e teve três números publicados.

$\mathrm{O}$ professor Chaim realizou diversas atividades administrativas em importantes instituições brasileiras. Atuou na direção e vice direção do IME - USP, por 16 anos, e na presidência do Instituto de Física Teórica por nove anos. Foi coordenador da Comissão de Pós-Graduação e da Comissão de Pesquisa do IME - USP e, ainda, membro do Conselho Universitário desta Universidade. Foi membro do Conselho Técnico-Científico do IMPA e coordenador da Assessoria de Matemática da CAPES, do CNPq e da Fundação de amparo à pesquisa do Estado de São Paulo - FAPESP. Foi, também, membro do Conselho Deliberativo do CNPq e do IMPA.

A atuação deste docente perante a direção do IME foi tão relevante que, no início da década de 1980, o influente professor Cândido Lima da Silva Dias, afirmou que o Chaim era "[...] a grande figura do instituto, o sustentáculo do instituto." (DIAS, 1981-84, p. 73).

Embora tenha assumido tantas atividades administrativas, a prioridade do professor Chaim sempre foi estudar Matemática. Mesmo nos períodos de atividades administrativas intensas, permaneceu investindo em sua carreira acadêmica, continuou lecionando, orientando e realizando pesquisas matemáticas.

De acordo com Silva (2008), o professor Chaim é reconhecido como um grande matemático na área de Análise e um importante líder para a Comunidade Matemática no Brasil. Corroborando esta ideia, o professor D'Ambrosio, em entrevista concedida a nós em 2009, afirmou que o professor Chaim "[...] tem uma Matemática muito boa, Análise Funcional, uma liderança muito forte na Matemática brasileira. Tem muitas publicações, bom professor, bom organizador" (informação verbal).

Neste sentido, com base nas informações obtidas com alguns acadêmicos entrevistados, podemos considerar o professor Chaim como um pesquisador que possuía uma visão ampla da Matemática, que conhecia todas as suas áreas de pesquisa.

Segundo D'Ambrosio, entrevista concedida a nós em 2009, o professor Chaim, além de ser "[...] um grande matemático é um homem muito culto, muito culto" (Informação 
verbal). De fato, este docente possuía um vasto conhecimento de línguas, era fluente em Português, Inglês, Frances e Alemão e ainda, lia Russo, Italiano e Espanhol.

Além disto, podemos destacar a paixão do professor Chaim pelos livros e pelas bibliotecas. A biblioteca de livros e revistas da área de Matemática do Professor Chaim, segundo a biografia apresentada no sítio da $\mathrm{ABC}$, era constituída por aproximadamente 15000 volumes. A esse respeito, o professor Chaim, entrevista concedida a nós em 2010, afirmou "A minha biblioteca era, até alguns anos atrás, provavelmente, fora a biblioteca do instituto [IME] e do IMPA, a melhor. [...]" (informação verbal).

Durante a sua carreira, em reconhecimento a sua atuação acadêmica, em 1955, foi eleito membro associado da Academia Brasileira de Ciências e, em 1968, tornou-se membro titular. Em 1998, foi agraciado com o título de Comendador da Ordem Nacional do Mérito Científico ${ }^{10}$, na área de Ciências Matemáticas.

Recebeu, também, o título de Associado Honorário da SBM ${ }^{11}$. Com relação a este prêmio, por ocasião do falecimento do Professor Chaim, Marcelo Viana, afirmou:

Chaim foi um pioneiro notável do desenvolvimento da Matemática brasileira, como primeiro presidente da SBM e coordenador do primeiro Colóquio Brasileiro de matemática. Sua eleição como associado honorário da SBM, que teve lugar durante meu mandato como presidente, foi um justo reconhecimento dessa trajetória singular ${ }^{12}$.

Manteve-se ativo até 2012, lecionando "cursos de verão" no IME e frequentando periodicamente a biblioteca deste Instituto, na qual acompanhava as publicações recentes da área de Matemática. Nesse período, aos 85 anos, dedicava-se ao estudo dos Fundamentos da Matemática. O professor Chaim, em entrevista ao Professor Possani, em 2003, afirmou que realizar pesquisas na área de Matemática após os oitenta anos não era nada excepcional, afinal ele concordava com José Reis, que afirmava a existência de duas atividades humanas nas quais a qualidade e o trabalho não diminuem com a idade: uma é a poesia e a outra é a Matemática.

Posteriormente, mudou-se para a cidade de Piracicaba-SP e faleceu no dia 19 de março de 2018. Chaim casou-se com Sônia Lohse (1939 - 2005), com quem viveu mais de 40 anos e teve quatro filhos Rebeca, Daniel, David e Raquel.

No quadro 1, a seguir apresentamos algumas fotografias deste docente.

\footnotetext{
${ }^{10}$ De acordo com decreto n. 4.115 de 2002, a Ordem Nacional do Mérito Científico "[...] tem por finalidade condecorar personalidades nacionais e estrangeiras que se distinguiram por suas relevantes contribuições prestadas à Ciência, à Tecnologia e à Inovação." (s/p)

11 Este é concedido a um acadêmico que o trabalho tenha "[...] contribuído de forma relevante para o desenvolvimento da Matemática no Brasil" (SBM, s/d, s/p)

${ }^{12}$ Na nota de Falecimento do Professor Chaim disponível no site da SBM.
}

RBHM, Vol. 19, n 37, p. 117-139, 2019 


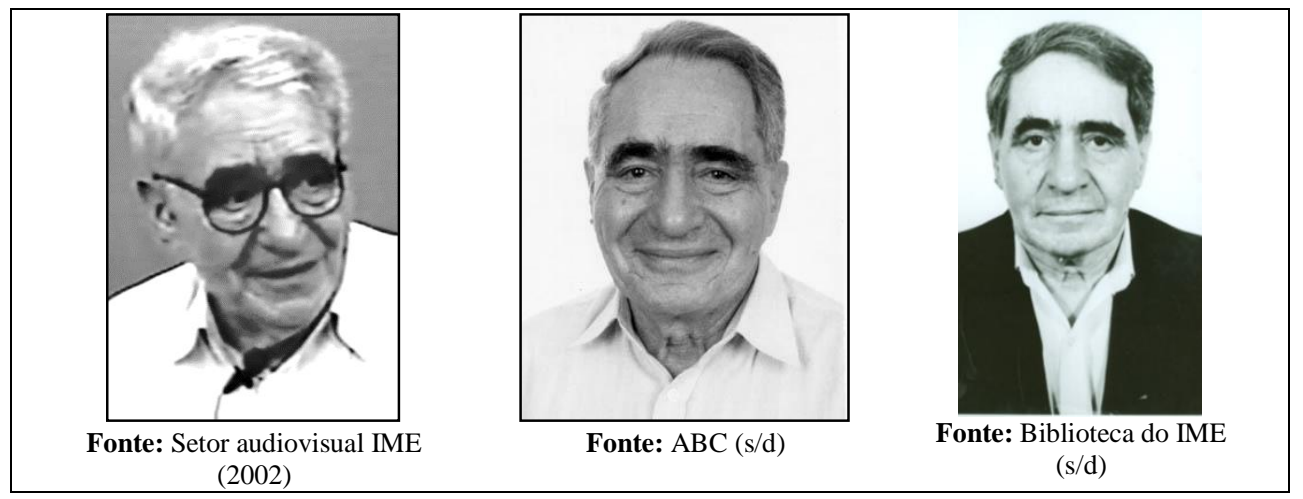

Quadro 1: Fotografias do Professor Chaim S. Hönig

Após a exposição de dados biográficos do professor Chaim e de aspectos de sua trajetória profissional, apresentaremos informações relativas à sua produção matemática.

\section{A pesquisa Matemática do Professor Chaim Hönig}

Os estudos e publicações do professor Chaim, conforme apontado, versavam primordialmente sobre Análise e Topologia. No entanto, pelos dados coletados, podemos perceber que em diversos momentos, o professor Chaim realizou, simultaneamente, estudos em diferentes áreas da Matemática.

De acordo com este professor, em entrevista concedida a nós em 2010, "Eu trabalhei em diversas áreas [simultaneamente] e quando tinha oportunidade e achava que o trabalho valia alguma coisa eu publicava... Eu nunca fui muito fã de publicar. [...] nunca me impressionei por número de publicações. Como os bons matemáticos brasileiros" (Informação verbal).

De fato, identificamos que os professores da FFCL da USP não tinham um grande número de publicações e isto pode ter uma relação com o fato de que neste período a Universidade brasileira ainda não estava impregnada do que se convencionou designar de "produtivismo acadêmico" no qual há uma supervalorização da quantidade de publicações de um professor universitário, em especial, da quantidade de artigos publicados em periódicos científicos.

Além disto, as obras do Professor Chaim são, de acordo com Fernandez, em entrevista em 2011, “[...] maduras, rigorosas, [...] resultado de um amadurecimento, de algo extremamente profundo". (Informação verbal). Corroborando a esta informação, o professor Ubiratan D'Ambrosio, em entrevista concedida a nós em 2009, afirmou que o professor Chaim produziu "[...] uma Matemática minuciosa, rigorosa, é difícil achar uma coisinha que está fora do lugar" (informação verbal).

No início de sua carreira, o professor Chaim, ainda na graduação, se interessou pelo estudo da Teoria dos Grupos, Teoria dos Reticulados e Topologia. Seu primeiro artigo publicado em um periódico científico foi na área de Teoria dos Grupos. O texto intitulado "Classificação dos Grupos Aditivos de números Racionais" foi publicado no Boletim da 
Sociedade Matemática de São Paulo, em 1951, e é considerado por Castro (1999) como uma contribuição interessante a esta área da Matemática. Este artigo, segundo Hönig (1959), inspirou um importante trabalho de Paulo Ribenboim (1928- ).

No início dos anos 1950, conforme já apontado, Chaim era assistente do professor Edison Farah na cadeira de Análise Superior e iniciou uma investigação no âmbito desta cátedra que resultou em sua tese de doutoramento denominada "Sôbre um método de refinamento de Topologias". Alguns resultados desta tese, foram publicados, em um artigo homônimo, no Boletim da Sociedade Matemática de São Paulo. Este artigo, foi considerado por Castro (1999), como uma contribuição significativa à Topologia Geral no Brasil. Além disto, de acordo com Hönig, em entrevista a Cláudio Possani em 2003, esta tese, posteriormente, foi integralmente publicada nos exercícios da segunda edição da obra "Topologia Geral" do grupo Bourbaki.

No período após o doutoramento, Chaim se dedicou ao estudo da Teoria dos Conjuntos e, em 1954, publicou no Proceedings of the American Mathematical Society, o artigo intitulado Proof of Well-ordering of Cardinal Numbers. Neste texto Hönig apresenta uma demonstração curta do Teorema da Bem Ordenação dos Números Cardinais, utilizando apenas o Teorema de Zorn (HÖNIG, 1959).

Simultaneamente, segundo Hönig (1959), Chaim realizou estudos na área de Álgebra e Geometria Algébrica, inicialmente no Brasil, juntamente com o professor L. H. Jacy Monteiro (1921-1975) e, posteriormente, na França no período do pós-doutoramento em meados dos anos 1950, quando teve a oportunidade de realizar, sob a supervisão de professor Marc Krasner (1912-1985), um seminário que abordava "Corpos de Valuações".

Ainda no pós-doutoramento, no Institut Henri Poincarè, em Paris, de acordo com Hönig (1959), Chaim participou de um curso lecionado pelo professor André Lichnerowicz (1915-1998) sobre Grupos de Holonomia, iniciando assim, seus estudos em Geometria Diferencial Avançada.

Posteriormente, no Brasil, dedicou-se ao estudo da Topologia Algébrica inicialmente com o professor Cândido Dias e posteriormente com o professor Eilenberg em São Paulo. Participou de um curso proferido pelo professor Koszul (1921-2018) que abordava, dentre outros assuntos, Teoria dos Feixes e Cohomologia, Complexos Topológicos e Espaços Fibrados. Estas influências se refletiram no capítulo intitulado "Topologia e Superfícies Compactas" do livro "Notas sobre Superfícies de Riemann", publicado pelo Departamento de Matemática da FFCL de São Paulo em 1957 (HÖNIG, 1959).

O professor Chaim, segundo Hönig (1959), também se interessou pelo estudo de Álgebras de Lie, participou de um seminário sobre este tema juntamente com diversos professores da FFCL de São Paulo e, posteriormente, assistiu a um curso do Professor M. Goto que versava sobre Grupos e Álgebras de Lie. Nos anos de 1956 e 1957, proferiu, no ITA, dois cursos. O primeiro abordava Teoria dos Grupos de Lie, no qual foram tratados Grupos não-Clássicos, Teoria dos Grupos Localmente Compactos e Teoria dos Espaços de Recobrimento. Já o segundo, versava sobre Álgebra Multilinear e Variedades Diferenciáveis e, introduzia a Teoria dos Grupos de Lie. Parte deste curso foi redigido, com o auxílio de Nelson Onuchic (1926-1999) e Leo H. Amaral (1926), para o primeiro Colóquio Brasileiro de Matemática. 
No final de 1958 e início de 1959, o professor Chaim, com base em alguns resultados já obtidos na área de Topologia, percebeu que poderia generalizar "de modo simples" a classificação de Kurosh, assunto que tinha se dedicado no início da década de 1950. Assim, produziu, em 1959, a tese para o concurso de provimento da cátedra de Complementos de Matemática da Faculdade Nacional de Filosofia, intitulada "Sobre uma generalização dos números Reais e sua aplicação na classificação de grupos sem torção". Alguns resultados desta tese foram divulgados, no início da década de 1960, nos artigos Sur les groupes sans torsion e Classification des groupes sans torsion, publicados em periódicos franceses (HÖNIG, 1959).

Embora tenha realizado estudos nos campos de Álgebra e de Topologia, a maior parte da produção acadêmica do professor Chaim é referente à área de Análise e Análise Funcional.

O interesse do professor Chaim por estas áreas, segundo Hönig (1959), se iniciou no período de 1949 a 1951, por intermédio do professor Delsarte, que o auxiliou nos estudos sobre Teoria das Distribuiçõos, Teoria das Integrações e Espaços Vetoriais Topológicos. Os estudos sobre estes espaços tiveram continuidade com o apoio dos professores Cândido Dias e A. Groetendieck (1928-2014).

No período do pós-doutoramento na França participou de dois cursos, um ministrado pelo Professor Jean Leray (1906-1998) que versava sobre Álgebras Localmente Convexas e outro lecionado pelo professor Laurent Schwartz sobre as Aplicações das Distribuições às Equações de Derivadas Parciais. Ao retornar ao Brasil o professor Chaim supervisionou Seminários de Especialização que abordavam problemas relativos a Estruturas Uniformes, Álgebras de Banach, Teoria da Integração e Espaços Vetoriais Topológicos (HÖNIG, 1959).

No final da década de 1950, iniciou seus estudos sobre Teoria das Funções Analíticas de mais de uma Variável e Teoria das Variedades Analíticas. Participou dos seminários da FFCL sobre esta temática e assistiu a um curso ministrado pelo Professor R. Gunning (HÖNIG, 1959).

Em meados da década de 1960, devido ao fato do professor Chaim ter assumido, como contratado, a regência da recém-criada cátedra de Equações Diferenciais, ele elaborou a tese intitulada "Análise de Fourier em espaços $\mathrm{L}_{2}$ e teoremas do tipo de Sobolev", para o concurso de Livre-docência desta cátedra, que reune resultados obtidos em diferentes momentos de sua carreira. (HÖNIG, 1965).

A partir da metade da década de 1960, conforme apresentado anteriormente, o professor Chaim passou a dedicar-se, em especial, ao estudo da Análise e da Análise Funcional e nos anos 1970, este docente tornou-se professor titular do IME - USP.

Com base nas informações apresentadas e em Silva (2008), podemos afirmar que os professores A. Groethendieck, L. Nachbin, L. Schwartz e Cândido Dias tiveram influência na formação matemática de Chaim. No entanto, enfatizamos que os dois últimos, foram citados por este acadêmico, em entrevista ao Professor Possani em 2003, como seus "mentores intelectuais", "exemplos a serem seguidos" (Informação verbal). 
Com relação as suas publicações, identificamos 8 artigos publicados pelo Professor Chaim em periódicos internacionais e 5 artigos em periódicos nacionais ${ }^{13}$. Além disto, o Professor Chaim publicou diversos livros voltados para o ensino da Matemática em nível superior.

Localizamos, nesta investigação, 16 livros e/ou monografias, produzidas pelo Professor Chaim. Estes materiais, com exceção de um, abordam temas relativos à Análise e, em geral, em suas introduções apresentam-se como materiais para serem utilizados em cursos de graduação ou pós-graduação. Entendemos que o interesse em produzir tais livros pode ter relação com a formação do professor Chaim que ocorreu em um período no qual, conforme já explicitado, havia uma escassez, em língua portuguesa, de livros destinados ao ensino da Matemática para o ensino superior.

Dentre estas publicações do professor Chaim, destacamos, com base em dados obtidos em algumas entrevistas, três livros, a saber: "Análise Funcional e Aplicações" (publicado originalmente em 1970 e reeditado pelo IME após duas décadas); “Análise Funcional e o problema de Sturm-Liouville" (elaborado para o VIII CBM, publicado pelo IMPA na coleção "Monografias de Matemática" em 1976 e, posteriormente, em 1978, após revisão e ampliação foi editado pela Edgard Blücher); "Aplicação da Topologia à Análise" (redigido para o III CBM, foi publicado, em 1961, pela UFPE na coleção "Textos de Matemática” e, posteriormente, em 1976 foi reescrito e editado pelo IMPA na coleção "Projeto Euclides"). No quadro 2, apresentamos ilustrações de duas destas obras.

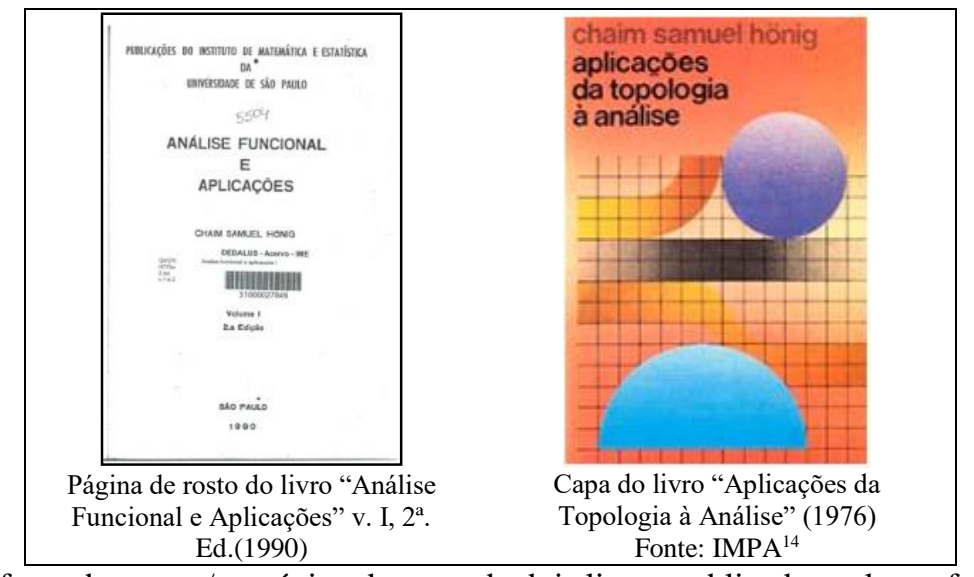

Quadro 2: fotos da capa e/ou página de rosto de dois livros publicados pelo professor Chaim

A alta qualidade destas publicações foi enfatizada por matemáticos entrevistados no decorrer da presente investigação. Além disto, estes acadêmicos mencionaram os livros redigidos pelo professor Chaim, como as produções acadêmicas mais relevantes deste

\footnotetext{
${ }^{13}$ Ressaltamos que o professor Chaim publicou alguns trabalhos sobre a História da Matemática no Brasil, mas estes não foram apresentados no presente artigo, por não se tratar especificamente da Matemática elaborada por este docente.

${ }^{14}$ Disponível em: https://impa.br/page-livros/aplicacoes-da-topologia-a-analise/.
}

RBHM, Vol. 19, nº 37, p. 117-139, 2019 
docente. Tais materiais foram amplamente utilizados para a formação de Matemáticos no Brasil. Neste sentido, estes livros foram citados em Silva (2008) e por acadêmicos entrevistados nesta investigação, como importantes obras no contexto da Matemática brasileira.

Após estas considerações a respeito da produção matemática do professor Chaim, apresentaremos algumas informações a respeito de suas contribuições para o desenvolvimento da Comunidade Matemática Brasileira.

\section{Algumas considerações sobre a atuação do professor Chaim para o desenvolvimento da Comunidade Matemática Brasileira}

Podemos, de maneira simplificada, com base nas ideias de Kuhn (1977, 1997), Kneller (1980), Fleck (1986), Oliveira (1989), Schwartzman (2001) e Bastos (2006), definir a Comunidade Matemática como um grupo de pessoas que se dedicam à pesquisa, à produção do conhecimento matemático. Estas pessoas estão em constante intercâmbio, por meio de sociedades, periódicos e eventos científicos. São, também, responsáveis pela formação de novos membros da Comunidade Matemática e, nesse processo de formação de novos membros elaboram e publicam manuais científicos.

Oliveira (1989) destaca que para uma "Comunidade Científica" se constituir é fundamental a existência de uma estrutura para a produção e socialização da ciência que seja constituída por: "a) instituições fortes e estáveis, para abrigar os grupos de pesquisa, o que demanda recursos; b) recursos humanos qualificados para exercer a atividade; c) canais de comunicação para fluir a produção científica." (p. 06).

Partindo destas ideias, teceremos considerações sobre a atuação do professor Chaim para o desenvolvimento da Comunidade Matemática Brasileira.

O professor Chaim, conforme já apontado, trabalhou na USP por mais de quatro décadas, atuando na graduação e na pós-graduação, inclusive em um período no qual a USP se configurava como um dos poucos centros de formação de matemáticos em território nacional.

Nesta Universidade criou seminários semanais, inicialmente, intitulados "Seminário de Análise Funcional e Equações Diferenciais", e posteriormente denominados "Seminário de Teoria da Integração e Equações Integrais". Estes seminários que foram criados na década de 1960 e perduraram por mais de três décadas, contaram com a participação de graduados e de pós-graduandos de diversas áreas de pesquisa em Matemática da USP.

A relevância da atuação do professor Chaim para a formação de matemáticos da referida universidade foi ressaltada por Fernandez, em entrevista concedida a nós em 2011, ao afirmar que "[...] muitas pessoas se formaram tendo aulas com ele, se formaram nos seminários dele [...]" (Informação verbal).

Além disto, encontramos registro de que o professor Chaim, orientou 15 pesquisas matemáticas de nível de mestrado e seis de doutoramento. Todos os matemáticos que foram orientados no doutoramento pelo professor Chaim seguiram a carreira acadêmica e atuaram em instituições de ensino superior no país, sendo que quatro destes orientaram pesquisas 
matemáticas em nível de doutorado, a saber: Nelson Onuchic, Toshio Hattori, Dicesar Lass Fernandez e Galdino Cesar Rocha Filho ${ }^{15}$.

Partindo do fato de que consideramos, inspirados por Coonce (s/d), que os descendentes de um cientista são os seus orientados de doutoramento, os orientados de seus orientados, nesse nível e assim por diante podemos afirmar que o professor Chaim possui descendentes científicos. Nesta investigação, identificamos 87 descendentes científicos ${ }^{16}$ do professor Chaim, sendo que alguns de seus descendentes, realizaram doutoramentos na área de Matemática e orientam investigações em outras áreas de pesquisa, como a Educação Matemática. Assim, localizamos descendentes científicos do professor Chaim que realizam e/ou realizaram pesquisas na área de Matemática (69 acadêmicos) ${ }^{17}$ e na área de Educação Matemática (21 acadêmicos) ${ }^{18}$. Neste sentido, enfatizamos que nem todos os descendentes acedêmicos do Professor Chaim são pesquisadores da área de Matemática.

Esses acadêmicos lecionaram e/ou lecionam em importantes instituições brasileiras, como, a USP, a Universidade Estadual Julio de Mesquita Filho (UNESP), Universidade Federal de São Carlos (UFSCar), Universidade Federal de Ouro Preto (UFOP), UNICAMP, Universidade Federal do Rio Grande do Norte (UFRN), Universidade Federal de Goiás (UFG) e Universidade Estadual de Londrina (UEL).

Além disto, merece destaque que alguns desses acadêmicos atuaram na criação de cursos de Matemática em importantes universidades no Estado de São Paulo, como o curso de Matemática da FFCL de Rio Claro, o curso de Matemática na UFSCar e a implementação do Bacharelado em Matemática na USP câmpus São Carlos (BADIN, 2006; UFSCAR, s/d).

Assim, com base nas informações apresentadas, podemos afirmar, de maneira análoga a Silva (2008), que os descendentes científicos deste docente têm expressiva atuação na pesquisa em Análise no Brasil.

Além disto, o professor Chaim, ainda, contribuiu para a formação de matemáticos brasileiros por meio da publicação de manuais científicos no Brasil. Publicou, conforme já apontado, vários livros destinados a formação de matemáticos brasileiros que versavam, primordialmente, sobre conceitos de Análise e Análise Funcional. A relevância destes materiais para o cenário matemático nacional foi ressaltada por acadêmicos entrevistados nesta investigação.

Merece destaque, também, que na década de 1950, foi realizada a primeira edição dos CBM que, conforme já indicado, previam a publicação dos textos que norteariam os

\footnotetext{
${ }^{15}$ Destacamos que a primeira orientação de doutoramento realizada pelo professor Chaim foi a do Professor Nelson Onuchic, na década de 1950 e a segunda foi concluída somente nos anos 1970. O distanciamento de mais de uma década entre as duas primeiras orientações desse docente pode, em parte, ser devido às dificuldades de um professor não catedrático da FFCL da USP orientar trabalhos desta natureza. Destacamos que o trabalho orientado pelo professor Chaim na década de 1950 é o único que versa sobre conceitos de Topologia, todas as outras pesquisas supervisionadas por ele são referentes a temas da Análise. Isto pode ter uma relação com o fato de, em meados dos anos 1960, o referido docente ter se tornado Professor Livre-Docente em Análise pela FFCL da USP. Conforme já apontado, percebemos situação semelhante com relação as publicações deste acadêmico.

${ }^{16}$ Dados relativos a julho de 2015.

${ }^{17}$ Destacamos que as orientações do professor Nelson Onuchic foram na área de Equações Diferenciais e, portanto, em um campo distinto de Topologia, o qual realizou o doutoramento sob a supervisão do professor Chaim. Aliás, o professor Chaim, também, não continuou suas pesquisas na área de Topologia conforme apontamos anteriormente.

${ }^{18}$ Estes totalizam 90 acadêmicos, sendo que três realizaram pesquisas em Matemática e em outra área.
}

RBHM, Vol. 19, nº 37, p. 117-139, 2019 
cursos proferidos durante o evento. De acordo com Lima (1995), muitos textos oriundos dos cursos proferidos nas primeiras edições dos CBM foram utilizados como referências em cursos de Matemática de várias instituições. Considerando que o professor Chaim foi o idealizador deste evento e que ele redigiu um texto para um curso do CBM, podemos afirmar que este docente contribuiu para a publicação de alguns dos primeiros manuais científicos no Brasil.

Tendo como referência essas informações podemos afirmar que o professor Chaim contribuiu para a formação de matemáticos na USP câmpus São Paulo e para a produção de manuais científicos que foram utilizados em diversos cursos de graduação e pós-graduação em território nacional.

Além disto, destacamos a relevância da atuação do professor Chaim para criação e ampliação dos canais de comunicação entre os Matemáticos Brasileiros.

Foi o idealizador e organizador do I CBM que, se configura como o primeiro evento científico periódico destinado exclusivamente a matemáticos no Brasil. Este encontro possibilitou o intercâmbio entre os matemáticos atuantes no Brasil ${ }^{19}$ e contribuiu para a formação de matemáticos em nosso país ${ }^{20}$. Possibilitou, também, de acordo com o relatório do I CBM, a discussão e elaboração de sugestões para o aumento das pesquisas matemáticas em território nacional, bem como, a elaboração de planos de estudos que seriam executados por matemáticos em diferentes localidades do Brasil. Neste sentido, podemos afirmar a relevância dos CBM para o desenvolvimento da Matemática brasileira nos anos 1950 e 1960 .

Além da sua atuação nos CBM, o professor Chaim, também, foi coordenador, juntamente com o professor Luiz Adauto da Justa Medeiros ${ }^{21}$, da primeira edição da "Escola Latino-Americana de Matemática” (ELAM) que foi realizada no IMPA, em 1968.

Atuou na diretoria da SMSP, sociedade que congregava acadêmicos provenientes de diversas instituições brasileiras, que havia sido criada na década de 1940 e que fora dissolvida em prol da SBM (TRIVIZOLI, 2008).

O professor Chaim também, participou da criação da SBM, que foi fundada em 1969 e tinha como parte de seus objetivos estimular a pesquisa e o ensino de Matemática no Brasil (SANTOS, 2016). Enfatizamos que o professor Chaim foi o primeiro presidente desta sociedade, fato que demonstra um prestigio entre os matemáticos brasileiros naquele período. Durante o seu mandato de presidente da SBM, foi publicado o primeiro número do Boletim

\footnotetext{
${ }^{19}$ Este intercâmbio foi importante para os matemáticos brasileiros, especialmente, para os jovens. Com relação a esta situação, D'Ambrosio, afirmou, em entrevista em 2009, que os matemáticos que estavam iniciando a carreira naquele período, praticamente, não se conheciam e tinham pouco conhecimento acerca das pesquisas que estavam sendo realizadas em território nacional.

${ }^{20}$ Este evento possibilitou que jovens matemáticos assistissem cursos de temas que, naquele momento, eram tendências da pesquisa em Matemática no Brasil. Esses cursos, de acordo com o relatório do I CBM, foram criados com o intuito de possibilitar o aperfeiçoamento de jovens matemáticos, de apresentar o estágio que a Matemática estava naquele momento e de indicar alguns problemas que eram atuais. Na década de 1950, no Brasil, não existiam disciplinas de pós-graduação e, assim, de acordo com Toledo (2008), os cursos do I CBM possibilitaram aos graduados o contato com a Matemática estudada após a graduação. Além disto, destacamos que os textos oriundos destes cursos, conforme já apontado, foram importantes para a formação de matemáticos em diversas instituições de ensino superior no Brasil.

${ }^{21}$ De acordo com Silva (2003), a coordenação do evento foi realizada pelo professor Luiz Adauto da Justa Medeiros. Conjecturamos que a coordenação científica ficou sob a responsabilidade deste docente, já que o coordenador da comissão local, de acordo com Hönig (1991), foi o professor Chaim S. Hönig.
} 
da Sociedade Brasileira de Matemática, do qual foi Editor-Chefe. Não encontramos, no desenvolvimento desta investigação, outro periódico brasileiro de circulação nacional, exclusivamente destinado a divulgação de pesquisas matemáticas, que estivesse em circulação neste período, já que os periódicos Summa Brasiliensis Mathematicae e Boletim da Sociedade de Matemática de São Paulo tinham saído de circulação em 1968 e 1966, respectivamente.

Com base nestas afirmações, podemos destacar a atuação do professor Chaim para a criação e ampliação de canais de comunicação entre os matemáticos brasileiros nos anos 50, 60 e 70 do século XX.

Merece destaque que no final da década de 1960 e início da de 1970, concomitantemente com o aumento dos pesquisadores em Matemática no Brasil, começaram a surgir eventos que congregavam acadêmicos de áreas específicas da Matemática. Na área de Análise, de acordo com Toledo (2008), foram realizados sete eventos que tiveram papel preponderante na constituição e automatização, no Brasil, desta área de pesquisa.

Dentre estes eventos citados por Toledo (2008), destacamos os seis que o professor Chaim participou da comissão organizadora, a saber: as três "Quinzenas de Análise Funcional e Equações Diferenciais" realizadas no ITA, o "Simpósio de Análise" ocorrido na UFPE, bem como, a "Semana de Análise Funcional Não-Linear" e o "Simpósio de Análise Funcional" realizados na UNICAMP.

Além destes eventos, o referido autor destaca que a inauguração dos Seminários Brasileiros de Análise (SBA), do qual o professor Chaim foi organizador por diversas vezes, foi extremamente relevante para a institucionalização desta área em território nacional. Neste sentido, ressaltamos, da mesma forma que Toledo (2008), a relevância da atuação do professor Chaim para o desenvolvimento da área de Análise no Brasil.

Com base nestas informações podemos afirmar que o professor Chaim teve atuação relevante para o desenvolvimento da Comunidade Matemática Brasileira e da área de Análise no Brasil. Afinal, este docente atuou na formação de novos membros para esta comunidade e na elaboração de manuais científicos, sobretudo da área de Análise, utilizados em diferentes instituições brasileiras. Atuou, também, na criação e ampliação dos canais de comunicação entre os membros desta comunidade, pois foi coordenador do I CBM, foi o primeiro presidente da SBM, o primeiro Editor-Chefe do Boletim de SBM e foi membro da comissão organizadora de vários eventos científicos que congregavam, em especial, analistas brasileiros.

\section{Considerações finais.}

Esta investigação foi realizada com o intuito de analisar a atuação acadêmica do professor Chaim Samuel Hönig para o desenvolvimento da Matemática no Brasil. Neste contexto, investigamos sua trajetória acadêmica privilegiando suas principais obras e suas contribuições para a criação e o desenvolvimento da Comunidade Matemática brasileira.

Os dados obtidos nesta investigação nos permitem afirmar que o professor Chaim, ao longo de sua trajetória acadêmica, se dedicou a pesquisa em várias áreas da Matemática, em especial, Análise e Análise Funcional. Dentre suas publicações destacamos os livros 
destinados a formação de Matemáticos brasileiros que versam sobre estas áreas da Matemática.

O professor Chaim, também, contribuiu para a formação de matemáticos brasileiros por meio de sua atuação, por mais de quatro décadas, na USP. Neste contexto, o professor Chaim orientou pesquisas de doutoramento e, atualmente, possui uma descendência matemática expressiva. Os acadêmicos que constituem tal decendência atuam e/ou atuaram em variadas instituições brasileiras e, alguns destes, auxiliaram a criação de importantes cursos de graduação em Matemática no estado de São Paulo.

Os dados desta investigação também nos permitem afirmar que o professor Chaim teve atuação destacada na criação e ampliação dos canais de comunicação entre os matemáticos brasileiros, tais como, os CBM, a SBM e variados encontros científicos da área de Análise. Neste sentido, enfatizamos a relevância da atuação do professor Chaim para o desenvolvimento da área de Análise no Brasil, bem como para o desenvolvimento da Comunidade Matemática Brasileira.

Para finalizar, destacamos que durante a realização desta investigação identificamos que a escrita de biografias de matemáticos que tiveram importante atuação no cenário nacional, de fato, traz elementos que contribuem para a escrita da História da Matemática no Brasil. Assim, enfatizamos a necessidade da realização de investigações com foco nas biografias de matemáticos que tiveram relevante atuação no cenário nacional, como por exemplo, Fernando Furquim de Almeida, Flávio Botelho Reis, Djairo G. Figueiredo, Francis D. Murnagham, Ruy Luis Gomes, José Morgado e Alfredo Pereira Gomes.

\section{Bibliografia}

ABC - ACADEMIA BRASILEIRA DE CIÊNCIAS. Biografia de Chaim Samuel Hönig. s/d. Disponível em: <http://www.abc.org.br/resultado.php3? codigo=chonig>. Acesso em mai. 2008.

AZEVEDO, A C. P., SILVA, C. P. "Mestrados e Doutorados obtidos no Brasil entre 1942 e 2004". s/d. In. <http://www.sbhmat.com.br/matematica.pdf.> Acesso em jun 2008.

BADIN, M. G. Um olhar sobre as contribuições do professor Nelson Onuchic para o desenvolvimento da Matemática no Brasil. Dissertação (Mestrado em Educação Matemática da Universidade Estadual "Júlio de Mesquita Filho"- UNESP- Rio Claro), 2006. BARONI, R. S.; NOBRE, S. R. "A pesquisa em História da Matemática e suas ralações com a Educação Matemática" In. BICUDO, M. A. V. (org.) Pesquisas em Educação Matemática: concepções e perspectivas. São Paulo: Editora da UNESP, 1999. p. 129 -136. BASTOS, T. R. A concretização do Abstrato: história da Institucionalização das Ciências Matemáticas no Brasil. Belo Horizonte: Argvmentvm, 2006.

BOSI, E. Memória e Sociedade: Lembranças de velhos. 2ª . Ed. São Paulo: Edusp, 1987. BRASIL. DECRETO No 4115, DE 6 DE FEVEREIRO DE 2002. Dispõe sobre a Ordem Nacional do Mérito Cientifica, e dá outras providencias, Brasília, DF, fev 2002.

Disponível em: < http://www.planalto.gov.br/ccivil_03/decreto/2002/D4115.htm >. Acesso em mar. 2018. 
CAlABRIA, A. R. Primeiro Colóquio Brasileiro de Matemática: identificação de um registro e pequenas biografias de seus participantes. Dissertação (Mestrado em Educação Matemática da Universidade Estadual "Júlio de Mesquita Filho"- UNESP- Rio Claro), 2010. Francisco Antonio Lacaz Netto (1911-1991): um estudo biográfico. Tese (Doutorado em Educação Matemática da Universidade Estadual "Júlio de Mesquita Filho"UNESP- Rio Claro), 2015.

CAMARGO, J. L. C. Entrevista por correio eletrônico. Recebido por mafeiteiro@yahoo.com.br em 13/04/2011.

CASTRO, F. M. O. A Matemática no Brasil. $2^{\text {a }}$. Ed. Campinas: Editora da UNICAMP, 1999.

CASTRUCCI, B. "Benedito Castrucci”. In. FREITAS, S. M. Reminiscências. São Paulo: Maltese, 1993.

COBRA, T. T. L. Carlos Benjamin de Lyra e a topologia algébrica no Brasil. Tese (Doutorado em Educação Matemática da Universidade Estadual "Júlio de Mesquita Filho"UNESP- Rio Claro), 2014.

COONCE, H. "The Mathematics Genealogy". s/d. Disponível em: <http://genealogy.math.ndsu.nodak.edu/>. Acesso em jan. 2011.

COLÓQUIO BRASILEIRO DE MATEMÁTICA. Relatório do Primeiro Colóquio Brasileiro de Matemática, 1957.

D`AMBROSIO, U. "Reminiscências do meu tempo de estudante na Maria Antônia." In. SANTOS, M. C. (org.) Maria Antônia: uma rua na contra-mão. São Paulo: Nobel, 1988, PP. $53-65$

. "Prefácio". In. SILVA, C. P. A Matemática no Brasil: História de seu desenvolvimento. $3^{a}$. Ed revista e ampliada. São Paulo: Edigard Blücher Ltda, 2003.

. Entrevista realizada por Mariana Feiteiro Cavalari em Rio Claro em 03 de março de 2009. Duração aproximada de uma hora.

DIAS, C. L. S. "Candido Lima da Silva Dias". Língua e Literatura: Revista dos departamentos de letras da Faculdade de Filosofia, Letras e Ciências Humanas da Universidade de São Paulo. Número comemorativo. Ano X, v. 10-13, 1981-84, p. 61-74.

DUARTE, P. C. X. Cândido Lima da Silva Dias: da Politécnica aos primórdios da FFCL da USP. Tese (Doutorado em Educação Matemática da Universidade Estadual "Júlio de Mesquita Filho"- UNESP- Rio Claro), 2014.

FERNANDEZ, D. L. Entrevista realizada por Mariana Feiteiro Cavalari na UNICAMP em 25 de janeiro de 2011. Duração aproximada de 75 minutos.

FLECK, L. La génesis y el desarrolo de um hecho científico: introducción a la teoria del estilo de pensamiento y del colectivo de pensamiento. Trad. Luis Meana e Angel G. de Pablo. Madrid: Alianza Editorial, 1986.

GOMES NETO, J. B. Entrevista por correio eletrônico. Recebido por mafeiteiro@yahoo.com.br em 02/03/2011.

HÖNIG, C. S. Análise de Fourier em Espaços $\mathbf{L}_{2}$ e Teoremas do tipo de Sobolev. Tese apresentada no concurso de livre-docência da Faculdade de Filosofia Ciências e Letras da Universidade de São Paulo, 1965. 
Euclides.

Aplicação da Topologia à Análise. Rio de Janeiro: IMPA, 1976. Coleção Projeto 1978.

Análise Funcional e o problema de Sturm-Liouville. São Paulo: Edgard Blücher,

. Análise Funcional e Aplicações. Vol. I, $2^{a}$. Ed. São Paulo: Publicações do Instituto de Matemática e Estatística da USP, 1990.

; GOMIDE, E. F. "História das Ciências Matemáticas". In. MOTOYAMA, S.; FERRI, M. G. (coord.). História das Ciências no Brasil. V. 1, São Paulo: Ed. Da USP, 1979.

Currículo Vitae de Chaim Samuel Hönig, pertencente ao processo funcional da USP, 1956.

. Memorial de Chaim Samuel Hönig, fornecido pelo próprio autor, 1991.

Entrevista realizada pelo Professor Possani, em 2003, no IME - USP. A temática da entrevista foi a biografia do professor Chaim. CD-ROOM. Setor audiovisual da USP.

. Entrevista realizada por Mariana Feiteiro Cavalari em São Paulo em 19 de maio de 2008. Duração aproximada de 70 minutos.

. Entrevista realizada por Mariana Feiteiro Cavalari em São Paulo em 13 de outubro de 2010. Duração aproximada de uma hora e meia.

Entrevista realizada por Mariana Feiteiro Cavalari em São Paulo em 02 de fevereiro de 2011. Duração aproximada de 75 minutos.

IME- INSTITUTO DE MATEMÁTICA E COMPUTAÇÃO. Lista de concluintes da pósgraduação em Matemática do IME - USP, 2005.

KNELLER, G. F. A Ciência como atividade humana. Rio de Janeiro: Zahar, São Paulo: Editora da USP, 1980.

KUHN, T. S. A Estrutura das Revoluções Científicas. São Paulo: Perspectiva, 1997.

. A Tensão Essencial. Trad. Rui Pacheco. Lisboa-Portugal: Edições 70, 1977.

LACOUTURE, J. "A história imediata”. In. LE GOFF, J.; CHARTIER, R.; REVEL, J. (orgs.). A história nova. São Paulo: Martins Fontes, 1998.

LIMA, E. L. "Os cursos dos Colóquios Brasileiros de Matemática". In. Matemática Universitária, n. 19, SBM, dez. 1995, pp. 01- 11.

NOBRE, S. Elementos Historiográficos da Matemática presentes em Enciclopédias Universais. Tese defendida para obtenção do título de Livre-Docente em História da Matemática. Universidade Estadual "Júlio de Mesquita Filho"- UNESP- Rio Claro, 2000.

OLIVEIRA, M. Canais formais de comunicação do conhecimento antropológico produzido no Brasil. Dissertação (Mestrado em Ciência da comunicação da Universidade Federal do Rio de Janeiro- UFRJ), 1989.

ROCHA FILHO, G. C. Entrevista realizada por Mariana Feiteiro Cavalari em São Paulo em 11 de maio de 2011. Duração aproximada de 3 horas.

RODRIGUES, A. A. M. Entrevista realizada por Mariana Feiteiro Cavalari em São Paulo em 07 de dezembro de 2010. Duração aproximada de 3 horas e meia. 
SANTOS, V. O. Uma história da Sociedade Brasileira de Matemática durante o período de 1969 a 1989: criação e desenvolvimento. Tese (Doutorado em Educação Matemática da Universidade Estadual "Júlio de Mesquita Filho"- UNESP- Rio Claro), 2016.

SBM - SOCIEDADE BRASILEIRA DE MATEMÁTICA. Associados Honorários e Beneméritos. Disponível em: <https://www.sbm.org.br/associados/associados-honorarios-ebenemeritos $>$. Acesso em: abr. 2018.

Nota de Falecimento: Chaim Samuel Honig (1926-2018). Disponível em:

<https://www.sbm.org.br/noticias/nota-de-falecimento-chaim-samuel-honig-1926-2018>.

Acesso em: mar. 2018.

SCHWARTZMAN, S. Um espaço para a Ciência: Formação da Comunidade Científica no Brasil. Brasília: Ministério da Ciência e Tecnologia. 2001. Disponível em: $<$ http://www.schwartzman.org.br/sitesimon/>. Acesso em jul. 2009.

SCHMIDT, B. B. "Grafia da vida: reflexões sobre a narrativa biográfica". História Unissinos, vol. 8, $\mathrm{n}^{\circ}$ 10, jul/dez 2004, p. 131-142.

SILVA, C. P. A Matemática no Brasil: História de seu desenvolvimento. $3^{\text {a }}$. Ed revista e ampliada. São Paulo: Edgard Blücher Ltda, 2003.

. Início e Consolidação da Pesquisa Matemática no Brasil. v. 98. Brasília: Gráfica do Senado Federal, 2008.

, AZEVEDO, A C. P. "História dos estudos de pós-graduação em Matemática no Brasil desde a segunda metade do século XX". 2005.

Disponível em: < http://www.canalciencia.ibict.br/pesquisa/0133-Historia-pos-graduacaomatematica-no-Brasil-seculo-XX >. Acesso em mar. 2018.

SOUTO, R. M. A. Mario Tourasse Teixeira: o homem, o educador, o matemático. Tese (Doutorado em Educação Matemática da Universidade Estadual "Júlio de Mesquita Filho"UNESP- Rio Claro), 2006.

TOLEDO, J. C. Uma História do processo de institucionalização da área de Análise Matemática no Brasil. Tese (Doutorado em Educação Matemática da Universidade Estadual "Júlio de Mesquita Filho"- UNESP- Rio Claro), 2008.

TRIVIZOLI, L. M. Sociedade de Matemática de São Paulo: um estudo históricoinstitucional. Dissertação (Mestrado em Educação Matemática da Universidade Estadual "Júlio de Mesquita Filho"- UNESP- Rio Claro), 2008.

UFSCAR - UNIVERSIDADE FEDERAL DE SÃO CARLOS. Fundadores dos cursos de Matemática da UFSCar. s/d. Disponível em:

< http://www.dm.ufscar.br/cursos/grad/fundadores.html > Acesso em fev. 2011

Mariana Feiteiro Cavalari
Instituto de Matemática e Computação da Universidade
Federal de Itajubá - UNIFEI - campus Itajubá - Brasil
E-mail: mfcavalari@ @unifei.edu.br
Sergio Roberto Nobre
Universidade Estadual Paulista “Júlio de Mesquita
Filho" câmpus Rio Claro
E-mail: sergio.nobre@ unesp.br

RBHM, Vol. 19, nº 37, p. 117-139, 2019 